\title{
Subacute necrotizing encephalomyelopathy of infancy and childhood
}

\author{
DAVID YASHON ${ }^{1}$ AND JOHN A. JANE \\ From the Laboratory of Neuropathology, Department of Neurology and Neurological \\ Surgery, University of Illinois, Chicago, U.S.A.
}

SYNOPSIS Subacute necrotizing encephalomyelopathy appears to be a distinct pathological entity $\stackrel{\sim}{\circ}$ occurring in infancy and childhood. The neurological manifestations are diverse but the course is $\vec{\sim}$ one of progressive deterioration. The aetiology is not known and no treatment is available. Histo- $\infty$ logically the lesion is characterized by multiple areas of necrosis and is remarkable because of a lack of glial or small cell reaction. There is a predilection for the brain-stem but the entire neuraxis may $\vec{c}$ be involved. The mammillary bodies are generally spared. Capillary proliferation and status spongiosus are seen either separately or more commonly in combination. Areas may be found in which no neural tissue is spared or a nucleus may selectively be involved with sparing of adjacent white matter, or occasionally only white matter is involved. In this account the twenty-first, twenty-second $\vec{\oplus}$ and twenty-third cases, one of which had a lesion in the mammillary body, are reported. Possible . aetiological mechanisms are discussed.

In 1951 Leigh reported an entity with certain pathological features that appeared to distinguish this disease from the large group of encephalopathies of obscure origin in infancy and childhood. The designation 'subacute necrotizing encephalomyelopathy' has subsequently been accepted by Blackwood, McMenemey, Meyer, Norman, and Russell (1963). Since 1951 cases have been reported by Feigin and Wolf (1954, three cases), Richter (1957, three cases), Ford (1960, one case), Reye (1960, four cases), Lewis (1962, one case), Tom and Rewcastle (1962, one case), Peterson and Alvord (1964, four cases), Namiki (1965, one case), and Eiben, Dooley, and Stowe (1965, one case). As in other encephalopathies in young people, the symptoms are diverse but in general are represented by progressive mental and neurological deterioration. In subacute necrotizing encephalomyelopathy focal random necrotic lesions are characterized by capillary proliferation and little cellular reaction. The aetiology is not known and treatment, except for supportive care, is not available. In the present communication the twenty-first, twenty-second, and twenty-third cases are reported and the previous 20 cases collected. The literature pertaining to this and related conditions is reviewed

'Present address: Department of Neurological Surgery Western Reserve University, Metropolitan Hospital, Cleveland, Ohio, U.S.A.

\section{CASE REPORTS}

CASE 1 R.F., a 12-month-old, white baby girl was well $\stackrel{2}{\Rightarrow}$ until 3 months of age when, following a mild upper respiratory infection, episodic non-projectile vomiting began after feeding. Her appetite remained poor throughout the remaining illness. She had been breast fed for the first one and one-half months of life. The patient was the product of a full-term, normal, spontaneous delivery. Birth weight was $6 \mathrm{lb}$. Until the onset of symptoms her development was normal, or even precocious. She had been able to roll over, smile, and almost sit up. At $3 \frac{1}{2}$ O months she weighed $10 \mathrm{lb}$. Subsequently she deteriorated and required gastric intubation for feeding and $\mathrm{O}$ tracheostomy because of an inability to clear secretions. One sibling, age 7, was alive and well, and there was no음 history of any similar disease in any member of the family. The patient was the daughter of a physician, so $\mathrm{N}$

malnutrition was probably not a consideration.
On examination the patient was cachectic, lying in a decerebrate posture. Harsh rales were heard throughout $\mathrm{W}$ the lungs. Ophthalmological examination was within normal limits. Cranial nerves were intact. The deep tendon reflexes were hyperactive in both lower extremities and ankle clonus was present. The plantar responses were $\stackrel{S}{\rightarrow}$ bilaterally extensor. Reflexes in the upper extremities were diminished. Grasp reflex was absent. The Moro ${ }^{-}$ reflex and sucking reflex were present. The fontanelle $\frac{\vec{D}}{\mathscr{D}}$ was not bulging nor was the head enlarged.

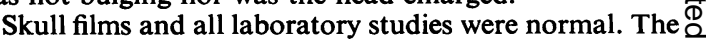
child died at 12 months of age. 
Necropsy Examination revealed bilateral bronchopneumonia and terminal gastric and duodenal ulcers.

Gross examination of the external surfaces of the brain revealed no abnormalities. On coronal sectioning the ventricular system was normal in size and configuration. There was a single haemorrhagic lesion in the left thalamus measuring $1.3 \mathrm{~cm} . \times 0.8 \mathrm{~cm}$. The spinal cord was removed and no lesions were seen.

HISTOLOGICAL EXAMINATION The brain and spinal cord were studied microscopically with the following methods: Kluver-Barrera, haemotoxylin-eosin, Weil, periodic acidSchiff, Van Giesen, Laidlaw, Perdrau, silver carbonate, and oil red-O.

Cortex The leptomeninges had their usual appearance except for areas, particularly over the frontal cortex, where there was prominent vascularity. Sections through the frontal, parietal, temporal, and occipital lobes showed normal myelination. The neurones and cortical layers were intact. In the callosal gyrus, however, there was a lesion involving both grey and white matter with increased capillary vascularity. These capillaries had thick walls with abundant collagen and reticulum and showed budding. The normal architecture was obliterated but no necrotic debris was present. Reactive glial elements were prominent, but there were no polymorphonuclear leucocytes or lipid macrophages. This was the only lesion in the cerebral cortex.

Thalamus Section through the thalamus showed a cavity filled with necrotic debris and haemorrhage. In the walls of the lesion lipid macrophages were seen, with intracellular pigment. Many capillaries were present and these were separated from one another by spaces. This lesion was atypical and is either different in aetiology or may merely represent a later stage of evolution (Fig. 1). No change was present within the subthalamic nucleus.

Hypothalamus An early subacute necrotic lesion was present within the borders of the mammillary body, spreading to the adjacent hypothalamus (Fig. 2). This lesion is early, but characteristic. The nuclear structure of the involved areas of the hypothalamus is disrupted although not entirely obliterated. The typical vascularity was present. Lesions were present in varying degrees of severity in adjacent areas of the hypothalamus bordering the third ventricle.

Midbrain Sections through the midbrain reveal multiple lesions similar to those described. In these areas neurones were relatively intact in spite of gross parenchymal destruction. Here also there was new capillary formation, dissolution of myelin, and astroglial and microglial proliferation. There was, in addition, status spongiosus. The aqueduct and peri-aqueductal grey matter was not involved and the necrotic lesions in the section of the midbrain were scattered with no particular relation to anatomical structures. The pineal gland was unaffected.

Pons A small lesion was present in the pons and is histologically similar to those described (Fig. 3).

Medulla At the level of the inferior olives a small characteristic necrotic lesion was noted in the floor of the fourth ventricle. The olives were entirely spared. Sections lower in the medulla at the level of the obex and decus- sation of the pyramids revealed necrosis of virtually all elements with status spongiosus. All tracts and nuclei were destroyed save occasional neurons. There was capillary proliferation and a great increase in glia. There was no haemorrhage, and there were no phagocytic cells present.

Cerebellum Sections at various cerebellar levels were normal.

Optic nerve No lesion was present in the optic nerves.

Spinal cord Sections through various levels of the spinal cord revealed no evidence of lesions either in the cord or in dorsal and ventral roots.

CASE 2 The illness in this 21-month-old, white baby girl (D.M.) apparently began at the age of 6 months, when the mother noted 'wandering' eyes and lagging development. The patient was admitted to hospital for investigation at the age of 16 months.

At that time her weight was 17 b. $11 \frac{1}{2} \mathrm{oz}$. She was $29 \frac{1}{2}$ in. in length. Head circumference was 18 in. The chest measured $18.5 \mathrm{in}$. and the abdomen 15 in. The anterior fontanelle was patent. The patient was underdeveloped and undernourished but appeared to be in no acute distress. The skin had a yellow, sallow appearance. The liver was palpable $3 \mathrm{~cm}$. below the right costal margin. Optic atrophy and horizontal nystagmus were present. Deep tendon reflexes in the lower extremities were hyperactive, and pinprick sensation was preserved. No positive toe signs were observed. She appeared to be severely retarded.

Birth weight was $6 \mathrm{lb} .9 \mathrm{oz}$. The mother had taken vitamin and iron supplements throughout gestation. Labour lasted for two-and-a-half hours and delivery had been normal and spontaneous. A sallow skin colour was the only possible abnormality which was noted at birth. The patient had been formula fed since birth, weighed $15 \mathrm{lb}$. at the age of 6 months, and had been followed in a well baby clinic. Meat was not taken well, so that iron and vitamin supplements were given. The baby had varicella, complicated by otitis media, shortly after the age of 6 months. She had had the full course of immunizations. The patient weighed $17 \mathrm{lb}$. at one year, and $21 \frac{1}{2} \mathrm{lb}$. at 20 months. She sat up at 10 months, crawled at 13 months. She had never walked. One sibling, aged 6, was normal; an uncle was 'spastic' but otherwise the family history was negative.

The clinical course was one of gradual deterioration. It was marked by diarrhoea, alternating with severe constipation, restlessness, anorexia, vomiting, hyperventilation, cyanosis, choking, and difficulty in feeding. The patient died at the age of 21 months.

Laboratory data Haemoglobin 12.6; haematocrit 43; W.B.C. 6,150 . Urine analysis was normal. Blood type was AB positive. Phenylpyruvic test of the urine was negative. Serum transaminase was 77 units. BUN was 16. Total serum cholesterol was $300 \mathrm{mg} \%$. Two hour postprandial blood sugar level was $111 \mathrm{mg} . \%$. Chest radiograph, skull films, and bone survey were normal. An E.E.G. was normal early in the disease. Electrolytes on several occasions revealed acidosis which was corrected by intravenous infusions. Liver function tests were normal except for a slightly elevated thymol turbidity. 


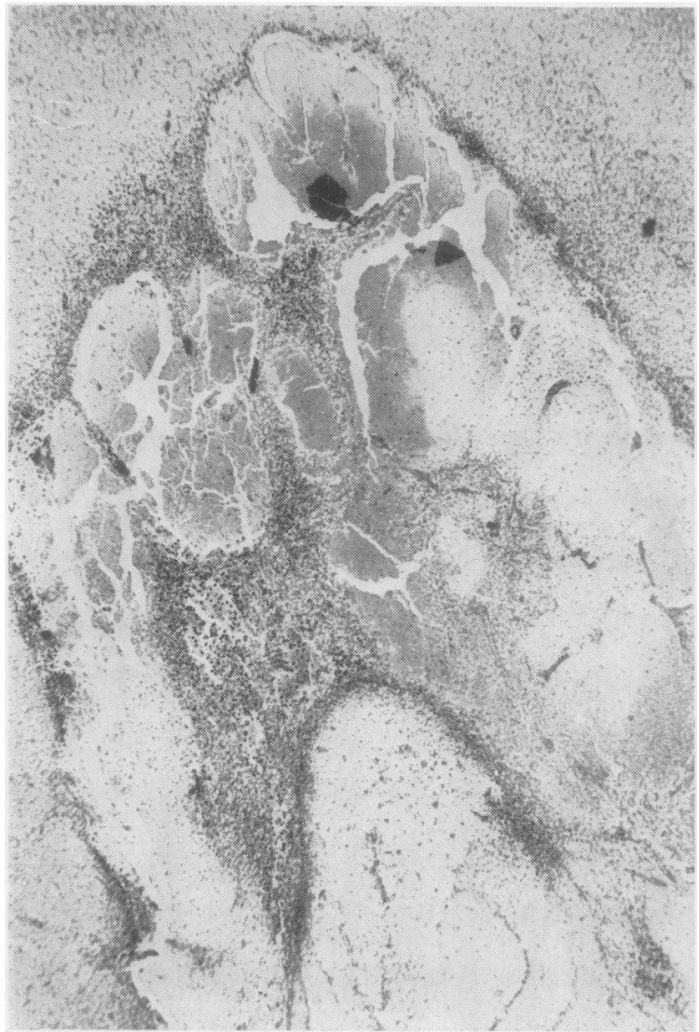

FIG. 1.

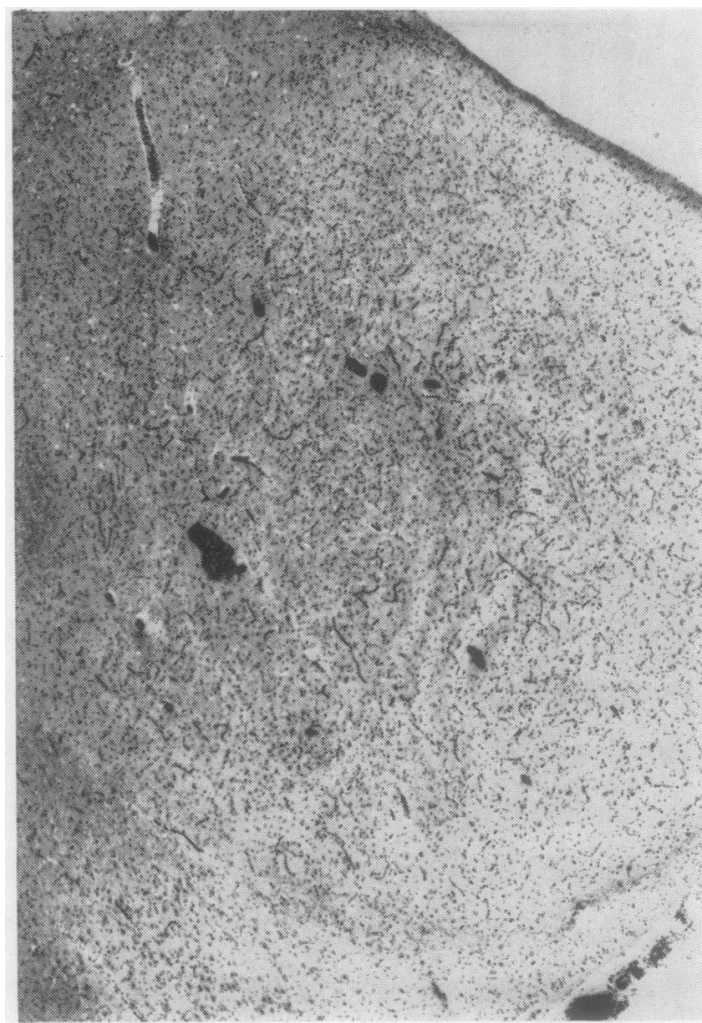

FIG. 2.

FIG. 1. Case 1. Thalamus. Cavitary area; possibly as a result of a more advanced lesion (Kluver-Barrera $\times 20$ ).

FIG. 2. Case 1. Mammillary body involvement $($ Weil $\times 40)$.

FIG. 3. Case 1. Pons and tegmentum. Sharp demarcation between involved and uninvolved areas $($ P.A.S. $\times 30)$.

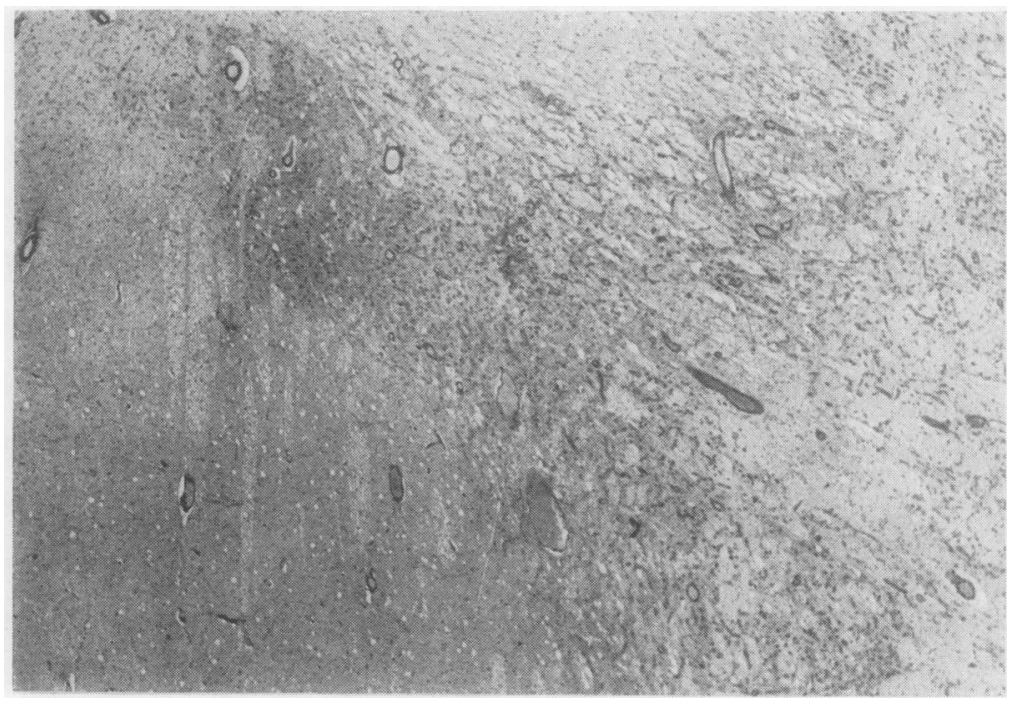


Total serum proteins were $7.0 \mathrm{mg} . \%$ (albumin $5.38 \mathrm{mg} . \%$ ) Serum electrophoresis revealed an elevation of globulin between the alpha ${ }_{2}$ and beta $a_{1}$ fractions.

NECROPSY Examination of the lungs revealed atelectasis and early bronchopneumonia. An easily reducible intussuseption measuring $1.5 \mathrm{~cm}$. was present in the upper ileum and the liver was congested.

The unfixed brain weighed $990 \mathrm{~g}$. No abnormality was noted grossly on the external surface. On coronal sectioning there were small cystic areas in the cerebellum and right cerebral peduncle.

HISTOLOGICAL EXAMINATION Various areas of the brain were sectioned and prepared using haemotoxylin and eosin, Kluver-Barrera, Weil, Perdrau, Van Giesen, periodic acid-Schiff, and oil red-O.

Cortex A moderate amount of diffuse increased capillary vascularity was present, but no discrete lesions were seen.

Thalamus and basal ganglia Sharply circumscribed lesions were present in the lateral geniculate body. There was striking proliferation of capillaries against a background of loose spongy stroma. Focal areas of necrosis with degenerative changes were present. No lesions were seen in the basal ganglia.

Midbrain The tegmentum had several lesions which were characterized by circumscribed areas of necrosis without cavitation. Spongy areas, capillary proliferation, and areas of capillary budding were present. Cellular reaction was minimal in that only a few lipid macrophages were present. There was no associated haemorrhage and the peri-aqueductal area was entirely spared.

Pons Small lesions were present throughout the pons and the middle cerebellar peduncle. Fibre tracts in these structures showed demyelination.

Medulla The medulla was strikingly involved (Fig. 4). Symmetrical and continuous, although irregular lesions, showing most of the features previously described were present, and here, as in other areas, there was no regard for either white or grey matter. The olivary nuclei were extensively destroyed.

Cerebellum Status spongiosus of moderate severity was present throughout the white matter of the cerebellum sparing the cerebellar folia. A small focal lesion was present in the white matter of the cerebellum. A striking and isolated lesion was seen in the dentate nucleus (Fig. 5). The ganglion cells were in various states of degeneration and compound granular corpuscles were present. In these lesions new capillary formation was not noted, but only degeneration of neurones and status spongiosus. The adjacent cerebellar white matter was uninvolved although a lesion was noted in the restiform body (Fig. 6).

Spinal cord Sections of the cervical cord showed generally symmetrical lesions with prominent involvement of the posterior columns. The anterior and posterior roots were not involved.

Optic nerves No characteristic lesion was seen although patches of poorly staining myelin were noted.

CASE 3 The illness of this 6-year-old, Negro boy (G.S.) commenced six weeks before death with the acute onset of focal left-sided seizures. The seizures were described as being tonic-clonic, and were followed by a profound state of lethargy. In the past history, both measles and chickenpox had been present several years earlier. The patient had been completely formula fed. He was delivered at home after labour lasting two hours and there were no perinatal difficulties. He had received all immunizations, the last being a 'triple' shot, administered three months before the onset of the illness. The family history was not significant.

On examination blood pressure was $98 / 70 \mathrm{~mm}$. $\mathrm{Hg}$. Pulse was 112 and regular. Temperature was $102 \cdot 2^{\circ} \mathrm{F}$. The patient could not be aroused and responded only to deep pain. A right central facial weakness was present. Eye examination was negative. The neurological examination revealed flaccidity of the trunk and limbs with absent deep tendon reflexes. Bilateral positive toe signs were elicited.

Seizures were controlled on admission with phenobarbital. Lumbar puncture showed a normal opening pressure while the cerebrospinal fluid protein was $16 \mathrm{mg} . \%$ and with no cells. Skull films were normal. The haemoglobin was $9.0 \mathrm{~g}$. upon admission.

While in the hospital the patient became less drowsy and he was able to eat with assistance. His condition then gradually deteriorated. While no further seizures occurred athetoid movements of the extremities began and continued with increasing frequency. The patient continued to deteriorate and expired, apparently due to bronchopneumonia.

The Mantoux test $(1: 1,000)$ was negative. Cerebrospinal fluid serology and gold curve were negative. Studies of the cerebrospinal fluid for fungi, acid-fast bacilli, and viruses were negative. Heavy metal studies were performed and were negative. A cause for the anaemia was not found.

NECROPSY The cerebral hemispheres were symmetrical and the meninges smooth and glistening.

On coronal sectioning after fixation the frontal and parietal lobes presented well-defined convolutions with good distinction between grey and white matter. Areas of dark grey and brown discoloration were present in both frontal lobes. These were almost entirely confined to the grey matter. On the left an abnormal area measuring $3.0 \mathrm{~cm} . \times 1.5 \mathrm{~cm}$. was seen and a similar area was present on the right. Similar lesions were present in the occipital lobes. No cavitation was noted. The ventricular system was normal in size and shape. The deep cerebral grey matter was spared of gross lesions but multiple punctate areas of discoloration were present in the pons and floor of the fourth ventricle. In the cerebellum, there were scattered similar discoloured areas.

HISTOLOGICAL EXAMINATION Sections of various areas of the brain were prepared according to periodic acid-Schiff, haemotoxylin and eosin, Weil, Kluver-Barrera, Van Giesen, Laidlaw, and Perdrau techniques.

Cerebral cortex Lesions throughout the cerebral cortex were plentiful, measuring about $2 \mathrm{~mm}$. in diameter, and involved predominantly the grey matter. Cortical 

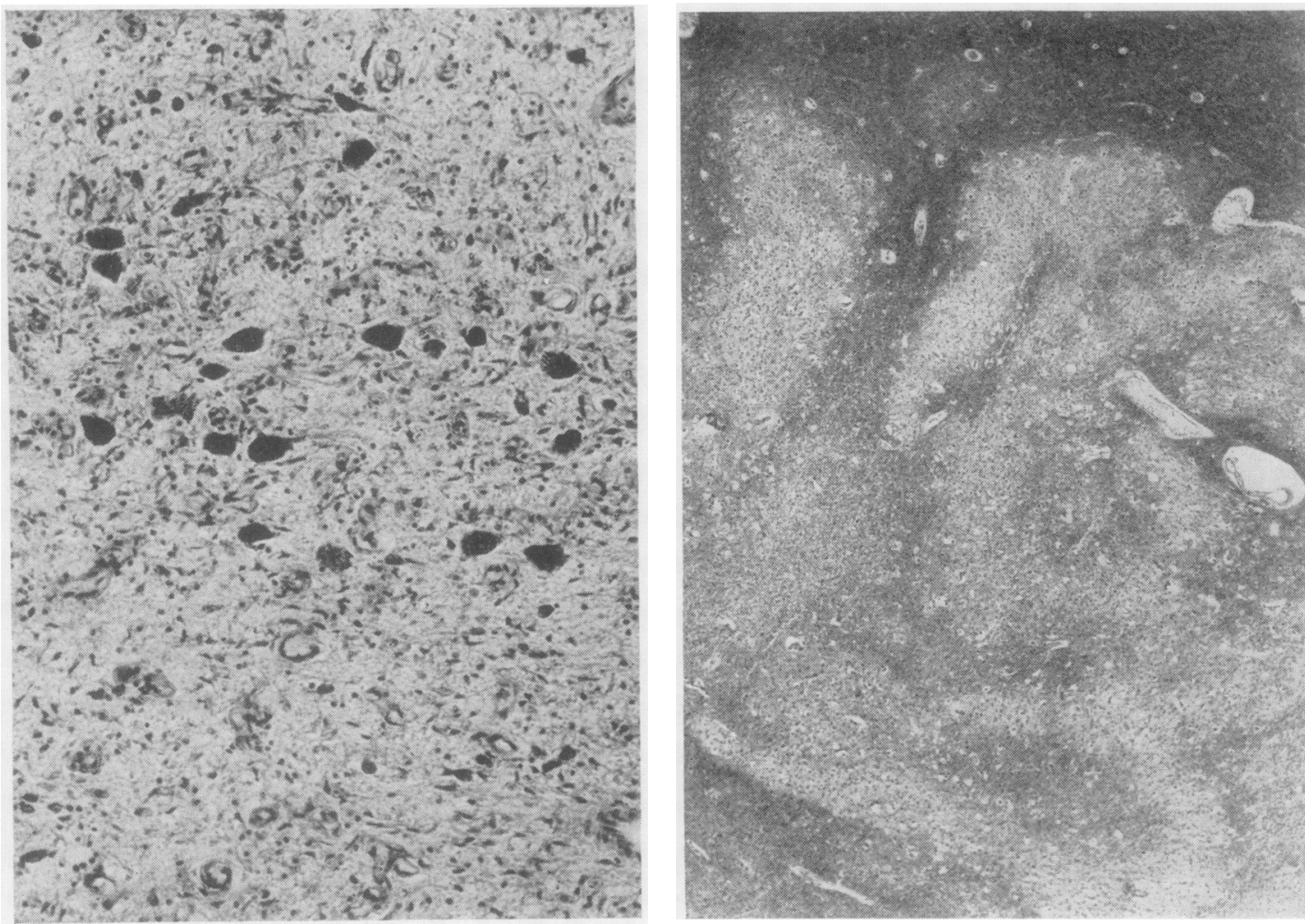

FIG. 4.

FIG. 5.

FIG. 4. Case 2. Medulla. Sparing of neurons within lesion (Kluver-Barrera $\times 120$ ).

FIG. 5. Case 2. Dentate nucleus. Selective destruction of dentate nucleus with sparing of adjacent myelinated areas No capillary hyperplasia $($ Kluver-Barrera $\times 26)$.

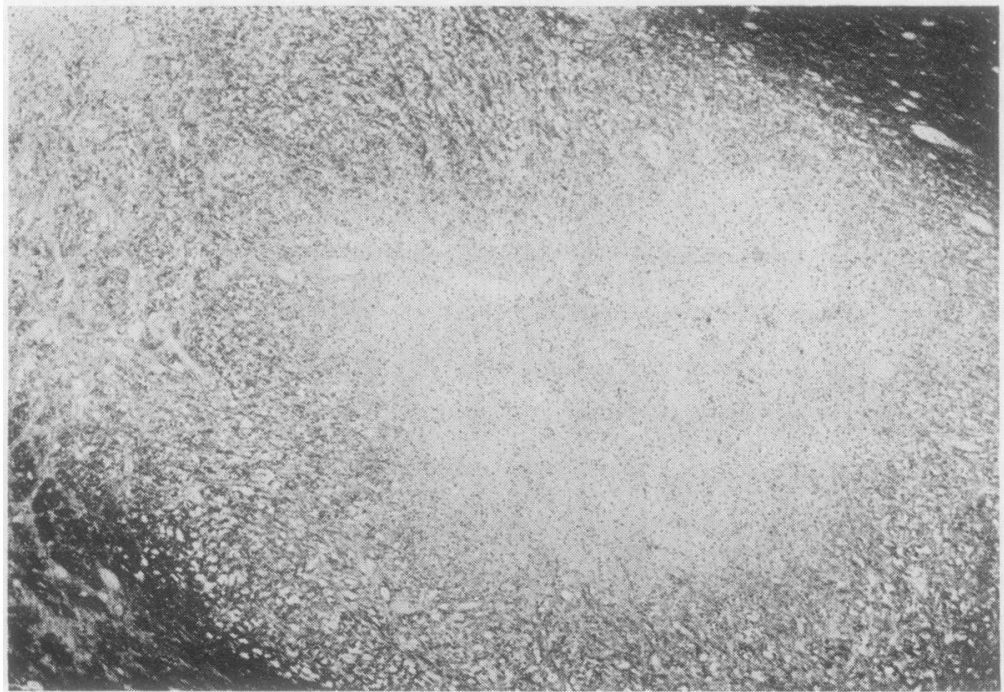

FIG. 6. Case 2. Restiform body. Demyelinization in centre, decreasing towards periphery of lesion $($ Weil $\times 30)$. 
lesions were characterized by capillary proliferation and these capillaries were thick walled (Fig. 7). A cellular reaction was absent. Neuronal elements were completely destroyed in areas of involvement. A sharp line of demarcation was present between involved and uninvolved areas. A relative status spongiosus was noted, in that the ground substance was much less dense as compared to normal areas. Only an occasional lipid macrophage was seen. There were no areas of necrotic debris or haemorrhage. The neovascular structures, which were the outstanding feature of these lesions, stained well with collagen stains but were most vividly seen with the periodic acid-Schiff method. Several areas in adjacent gyri were involved as if spread of the histological process had occurred by contiguity (Fig. 8).

Thalamus and basal ganglia Sections through the thalamus revealed several lesions similar to those previously encountered (Fig. 9). Here, however, small cell infiltration was more common and compound granular corpuscles were seen. Status spongiosus was apparent. Structures within the areas involved were completely destroyed. Similar involvement was present in the corpus striatum, internal capsule, and caudate nucleus. Nolesion was seen either in the hypothalamus or mammillary bodies.

Midbrain Sections through the midbrain at various levels revealed several subacute necrotic lesions. The periaqueductal grey matter was not involved. At this level the lesions contained conspicuous numbers of lipid macrophages but, otherwise, the lesions were identical to those described previously. Areas immediately adjacent to the lesions were normal without any reaction whatever.

Pons In the pons an earlier lesion was present without vascular or cellular reaction. Some glial proliferation was present but the spongy appearance was the main feature.

Medulla The floor of the fourth ventricle was studded with early lesions similar to those described in the pons as well as later lesions with vascular and glial proliferation. The olivary nucleus was entirely and selectively spared. This was in contrast to case 2 , where the nucleus was selectively destroyed with preservation of surrounding structures.

Cerebellum Many small areas of the cerebellum were involved without respect for grey or white substance or anatomical structures. No lesion was present in the dentate nucleus. The lesions present in the cerebellum were of the combined variety. Again, lesions were present in several areas of adjacent folia as if spread had occurred by trans-sulcal contiguity (Fig. 10).

\section{DISCUSSION}

CLINICAL ASPECTS The widespread dissemination of lesions in this condition accounts for the multiple signs and symptoms as well as for the only common feature, mental deterioration. Although there is some predilection for the brain-stem, lesions have been found throughout the nervous system, including demyelination in the peripheral nerves (Reye, 1960; Namiki, 1965). The mammillary body has been implicated only in our case 1 and in the case of
Eiben et al. (1965). This is noteworthy in view of the frequent involvement of the mammillary bodies in Wernicke's disease. The progression of the disease is slow but unrelenting.

These patients show feeding difficulties, lagging development, or regression of that development which had once been attained. Lethargy, irritability, and visual disturbances are frequently noted. In our case 3 , the admitting complaint was intractable generalized seizures. Seizures have occurred prominently in only two other cases (Richter's case 1, 1957; Eiben et al., 1965) and in those instances no cortical lesions were seen.

In keeping with the diverse lesions no characteristic signs are present although developmental retardation is common to all cases. Varying levels of consciousness, masked facies, excessive salivation, blindness with optic atrophy, deafness, spasticity, flaccidity, ataxia, athetosis, tremor, nystagmus, positive toe signs, and decerebration have all been noted. However, papilloedema and other phenomena indicative of increased intracranial pressure have not been seen. In six cases the results of the lumbar puncture are available. All were normal with the exception of a cerebrospinal fluid protein of $83.6 \mathrm{mg} . \%$ in the case of Tom and Rewcastle (1962), $67.5 \mathrm{mg} . \%$ in our case 2, and $100 \mathrm{mg} \%$ in one of the cases of Peterson and Alvord. Other diagnostic tools were not helpful. Skull films revealed no abnormality and E.E.G.s showed either varying degrees of non-specific abnormalities or were normal. Copper studies were normal in Peterson and Alvord's case (1964). In most cases complete antemortem study, including evaluation for viral disease, was normal.

It has been mentioned that, in spite of the chronicity of the disease, these patients sometimes are well nourished at the time of death (Richter, 1957). This was true in Richter's cases and in one of ours. However, two of our cases and at least one of those of Feigin and Wolf were cachectic when evaluated late in the course of the disease.

An analysis of the prenatal period in the 22 cases under discussion failed to reveal any consistent features that might be considered of aetiological significance. A secondary anaemia occurred in Richter's case 1, and the mother had a 'virus' infection in case 3 of Feigin and Wolf (1954). A threatened abortion occurred during the second month of pregnancy in Richter's case 2, and in Namiki's case the mother's prenatal nutrition was poor. In all cases the delivery was normal and full term. Trauma was not a factor and no perinatal hypoxic episode is reported. From the analysis of such prenatal and perinatal factors, no clues as to aetiology were obtained. 


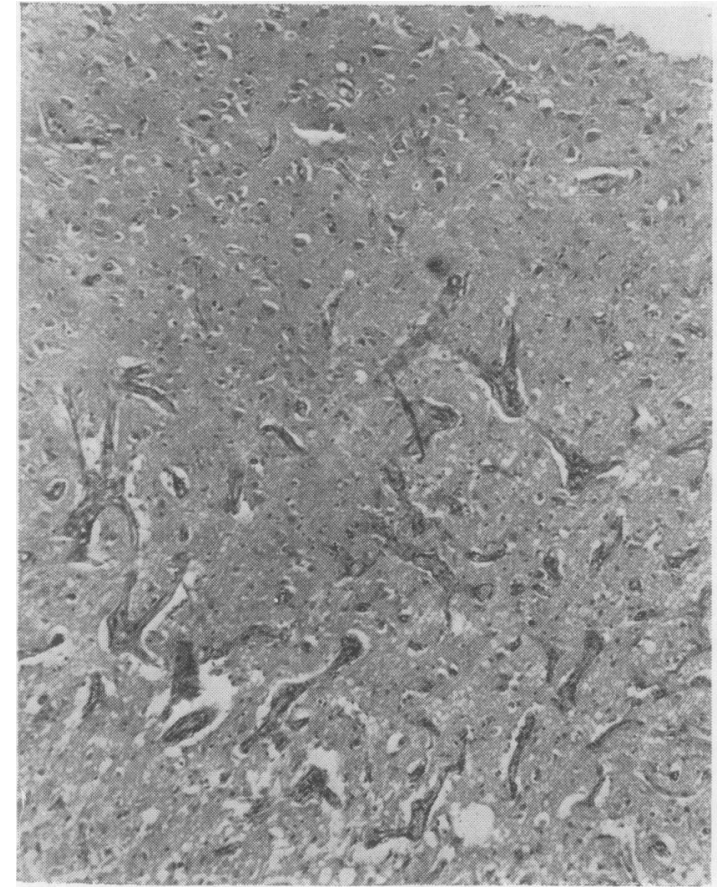

FIG. 7.

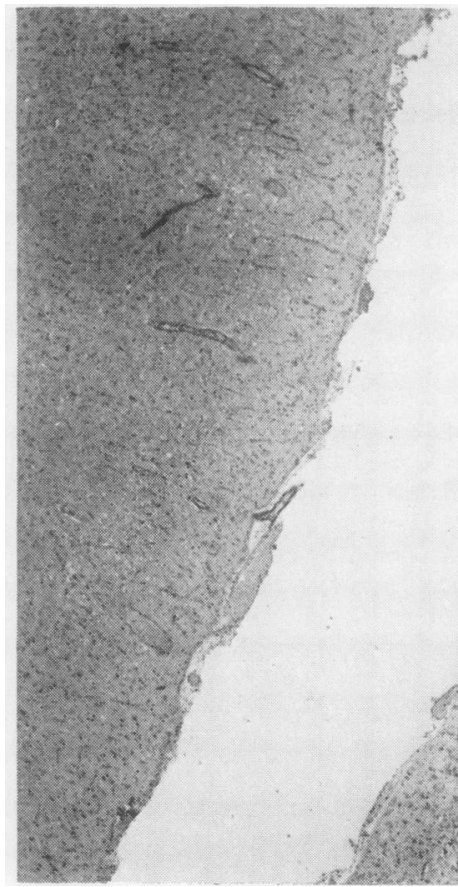

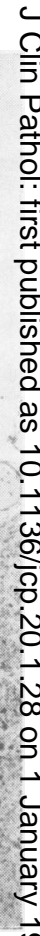

FIG. 7. Case 3. Cortex: areas of vascular and spongy involvement are separate (P.A.S. $\times 80)$.

FIG. 8. Case 3. Cerebral cortex: spread by trans-sulcal contiguity in adjacent gyri (Kluver-Barrera $\times 24)$.

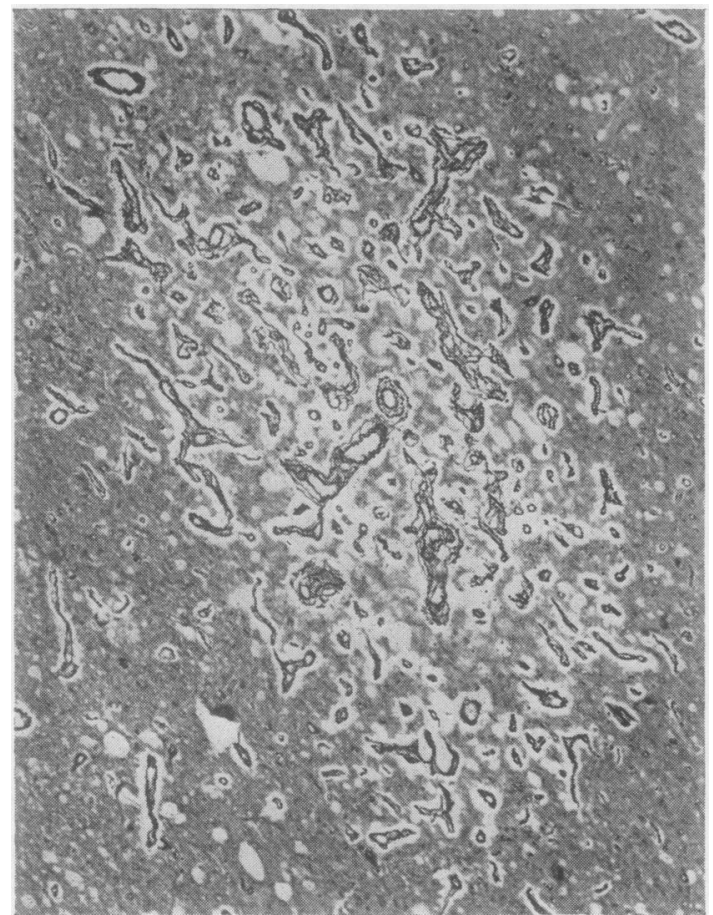

FIG. 9.
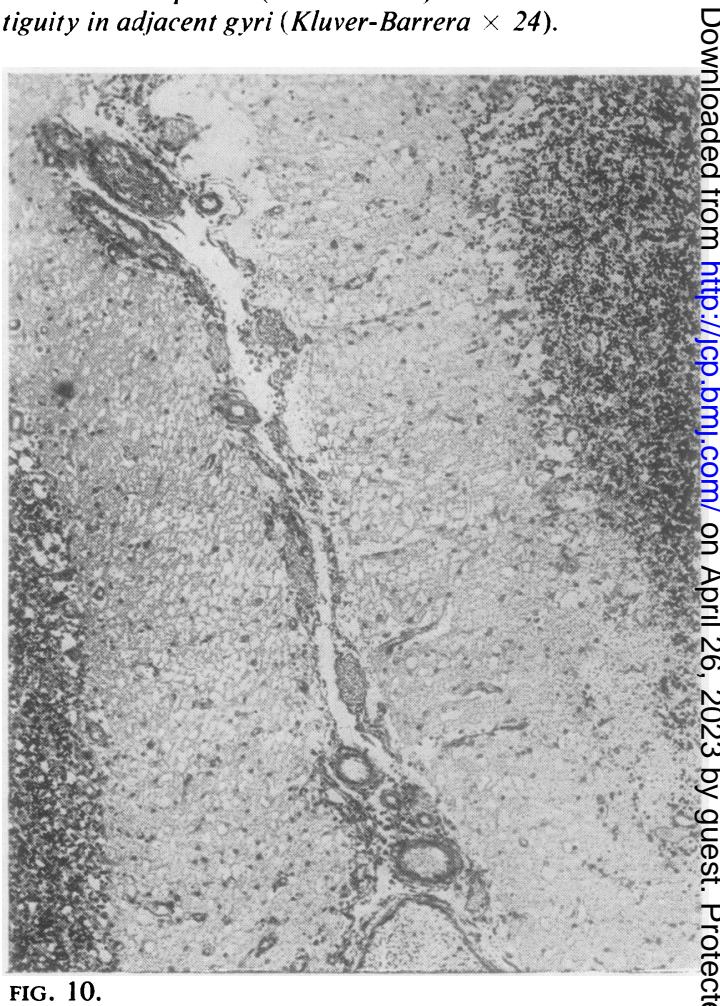

FIG. 9. Case 3. Thalamus: sharply demarcated area of involvement; capillary hyperplasia with increased reticuli@

$($ Perdrau $\times 60)$. $\quad$ O FIG. 10. Case 3. Cerebellum: spread by trans-sulcal contiguity in adjacent folia; spongy lesion in molecular layer granular layer is involved (haemotoxylin-eosin $\times 70$ ). 
The postnatal course has also in general, been unremarkable, although Richter's case 3 had feeding difficulties dating from birth. In the postnatal period feedings restricted to mother's milk have been implicated as a possible aetiological factor, since in so-called 'breast milk intoxication' (Tanaka, 1934) the lesions resemble those in subacute necrotizing encephalomyelopathy. At least five of the 22 patients were breast fed for variable periods. Three were not breast fed at all, and in the remainder information is not available. Since several patients were not breast fed and still had characteristic lesions, this factor is probably not important.

If we do not include Richter's third case which may have begun at birth, the youngest apparent age of onset was case 1 of Feigin and Wolf (1954), and the case of Lewis (1962) in which the disease was first noted at 2 months of age. Namiki's case was retarded from early infancy but died at age 15 . Peterson and Alvord (1964) have divided their cases into the subacute infantile form (which corresponds to all the cases thus far discussed except our case 3, Peterson and Alvord's three older cases, and Namiki's patient), and a chronic juvenile familial form. In the latter category the course of the disease is over several years and death may be as late as the early second decade. Although our case 3 began abruptly at age 6, the course was only of six weeks' duration so that placement into Peterson and Alvord's classification is difficult.

The length of active illness varied from a few days, as in Tom and Rewcastle's patient, to as long as three years, as in the third patient of Feigin and Wolf (1954). Chronic juvenile cases had courses lasting several years.

In many cases, there was a high incidence of perinatal deaths in the family history and there have been two instances of proven familial incidence. Thus cases 2 and 3 of Feigin and Wolf, and cases 2, 3, and 4 of Peterson and Alvord were siblings. In five other cases there were miscarriages, neonatal deaths, or deaths from a similar disease but without post-mortem examination. The parents of both the patients of Namiki and Feigin and Wolf were first cousins. The case of Eiben et al. had a sister who died of a similar pathologically verified disease. In our cases there was no pertinent family history and from the above evidence the importance of genetic factors, if any, cannot be determined. The fact that three families were definitely involved, and at a considerable time interval between cases, should arouse some suspicion of a significant constitutional factor. The high incidence of early deaths among siblings may also be of significance in this regard.

Two further reports where exact classification is difficult, but where obvious similarities to subacute necrotizing encephalomyelopathy are seen, should be briefly discussed.

Thus the two cases which were reported by Ule (1959) may fall into the category of subacute necrotizing encephalomyelopathy. The children died at ages $2 \frac{3}{4}$ and $4 \frac{1}{2}$ years, after a chronic neurological illness marked by progressive deterioration. Histological examination of the brain revealed lesions in the brain-stem similar to those found in the cases discussed here. Nutritional deficiency was not a known factor in these cases, although the author classified this illness as a 'Wernicke-like' pseudoencephalitis.

Verhaart (1938) studied the cases of four Chinese infants, all under 8 months of age, in which the lesion was similar. In the caudate nucleus, putamen, and globus pallidus, a generalized rarifaction of tissue occurred. Neurones were scanty to absent in the involved areas and there was an increase in capillary vascularity.

THE NATURE OF THE LESION The characteristic of this disease, which is unifying and without which classification as a separate entity would be impossible, is the histological appearance of the lesion. Two main aspects of the lesion may be discussed. The first is the prominent capillary vascularity. These abnormal capillaries are thick walled with abundant and varying amounts of reticulum and collagen. In spite of the very active capillary proliferation and necrosis there is generally little cellular reaction. In Reye's case 3, perivascular cuffing, suggestive of viral disease, was noted. Massive haemorrhage and thrombosis does not occur.

'Status spongiosus' is a second prominent component of this lesion. Breakdown of ground substance occurs so that a characteristic loose appearance develops. Here also, there is little cellular reaction and even in older lesions gliosis is usually not seen.

Abnormal vascularity and spongy stroma usually, but not invariably, are found together (see Fig. 7). The earliest tissue alteration is a spongy state in which the presence of a lesion may be questioned. The capillary component may then be superimposed upon the spongy component. The final lesion is the microscopic cavitation which occurred in our cases 1 and 3 (Fig. 1) and may be the lesion as represented in its most advanced state. Abundant neovascularization within the cavitations themselves, without surrounding neovascularity, supports this sequence. However, in the cases of Namiki and Peterson and of Alvord of many years' standing, no cavitations are mentioned and this may be used as evidence against such a progression.

Richter has stated that the preservation of 
neurones within areas otherwise almost completely destroyed was characteristic of the process. We have confirmed this finding in what we consider to be the early lesions (Fig. 4).

Another aspect of this pathological process is the varied involvement of parenchymal structures. The more common later lesion destroys white and grey matter without predilection for either. Thus, in the brain-stem almost all areas in a single cross section may be involved. However, the lesion may also be restricted to the periventricular grey matter or the cortical grey matter as in our case 3. This is in distinct contrast to the first type of lesion where myelinated areas are severely destroyed. Again only white matter may be involved with sparing of the grey. The third anatomical form, and that which is by far the least common, is the restriction to only a certain nuclear mass with sparing of adjacent and intervening areas. An example of this type of process occurred within the dentate nucleus of our case 2 .

Another interesting aspect of the histology of this disease is the apparent spread across the pia arachnoid so that lesions may be seen separated only by subarachnoid space. This spread by contiguity is obviously suggestive of some chemical influence.

RELATIONSHIP TO WERNICKE'S DISEASE, 'BREAST MILK INTOXICATION', AND CHASTEK PARALYSIS Wernicke in 1882 described what he called 'acute haemorrhagic polioencephalitis'. This description may be at least partially incorrect due to frequent involvement of white matter. The characteristic histological lesion in subacute necrotizing encephalomyelopathy has been likened to those present in Wernicke's disease. In Wernicke's disease the aetiological factor, presumably thiamine deficiency, is said to be related to some degree of inanition (Meyer, 1944). There are points of similarity between Wernicke's disease and subacute necrotizing encephalomyelopathy which may be discussed.

The most frequent neurological signs and symptoms in Wernicke's disease are due to involvement of the third nerve nuclei. This is not true of subacute necrotizing encephalomyelopathy where symptoms are variable. However, diverse neurological signs and symptoms may occur in either disease.

Another difference is the age of onset. In Wernicke's disease adults are usually affected, whereas subacute necrotizing encephalomyelopathy is restricted to infantile and juvenile age groups. However, in Campbell and Biggart's (1939) series of 12 cases of Wernicke's disease, one patient was $3 \frac{1}{2}$ years of age. The course of the entire illness was only three days. This patient had been breast fed for three months (Campbell and Russell, 1941). Two other siblings had previously died of a clinically simila disease without post-mortem examination. OM post-mortem examination of this child, the manas millary bodies and hypothalamus were heavił involved. In the study of Riggs and Boles (1944) of 42 cases of Wernicke's disease, one patient was age $2 \frac{1}{2}$ years. The history in this case was characterized by coma, convulsions, involuntary movements, and ophthalmoplegia. No history of gastrointestinal disturbance was present. Lesions were present in the cortex, mammillary bodies, thalamus, and peris aqueductal grey matter. Perhaps these childhoo cases of Wernicke's disease are more correctif grouped with subacute necrotizing encephalq5 myelopathy.

All cases of adult Wernicke's disease are said tio have a primary nutritional disturbance. This is if contrast to subacute necrotizing encephalomyeloo pathy where the nutritional state of some patients at the termination of the illness is often good (Richtes 1957). In the adult with Wernicke's disease a nutret tional deficiency is important and correcting this deficiency may reverse the course of the disease.

The lesion in Wernicke's disease is characterized by vascular stasis, petechial haemorrhages, parenchymatous degeneration, glial proliferation, and pro liferation of capillaries. The frequently seen sma haemorrhages which occur are a histological poigt of differentiation between Wernicke's disease and subacute necrotizing encephalomyelopathy, where such haemorrhages are rare.

Lesions in the mammillary bodies are almost cor stantly present in Wernicke's disease. Riggs an Boles (1944) examined the mammillary bodies in series of 23 cases of Wernicke's disease. Lesions this nucleus were present in 21 of the 23 patients. all 12 cases considered by Campbell and Bigga? (1939) the mammillary bodies were involved. On the contrary, there is only one previous report of sub acute necrotizing encephalomyelopathy where $\vec{a}$ lesion was reported in the mammillary bodies (Eiben et al., 1965). Our case 1 had an early lesio within the mammillary body and more advance lesions in adjacent areas. Although a difference emphasis on locations exists in these two diseases overall similarities are evident.

Considerations of similarities and differences between these two diseases may be important, sinee the lesions in each may be similar in pathogenes In the comparison of the two diseases, clues as the aetiology of subacute necrotizing encephalomyelopathy may be disclosed, especially since it is very probable that the lesions in Wernicke's disea are due to thiamine deficiency.

In 1934 (Tanaka, 1934) the histological findings $\stackrel{\mathbb{P}}{\stackrel{9}{2}}$ a case of so-called 'breast milk intoxication' we 
described. The syndrome consisted of progressive neurological deterioration with varied signs and symptoms which were seen only in breast-fed infants and was not uncommon in Japan during that period. When other sources of milk were given the infants uniformly recovered. The case described was that of a $5 \frac{1}{2}$-month-old boy whose illness lasted a total of four days. The histological findings revealed subacute necrotizing lesions with capillary proliferation in the cervical spinal cord, medulla, cerebellum, corpus striatum, and apparently in the cerebrum. Lesions in the mammillary bodies were not noted. Cruickshank (1950), in analysing the problem, felt that these cases were presumably due to infantile beri beri since, at that time, vitamin deficiency diseases were common among Japanese nursing mothers. From the solitary case report (Tanaka, 1934), it was not possible to ascertain whether this histological lesion resembles Wernicke's disease or subacute necrotizing encephalomyelopathy. The relationship of the lesions in so-called breast milk intoxication to those lesions in the two diseases under consideration is obvious. Since the lesions in all three diseases are similar, perhaps a similar class of aetiological agents might be invoked as being the causative agent in subacute necrotizing encephalomyelopathy. Here, rather than being a result of a nutritional deficiency as such, perhaps an enzymatic inability to utilize available vitamins is present. Further support of this notion may be gained by a consideration of 'Chastek paralysis' in foxes. The lesions in this condition are similar to Wernicke's disease and the aetiology appears to be thiamine deficiency (Evans, Carlson, and Green, 1942). This condition can be brought about by feeding fresh fish to foxes. The mechanism is not clear, but enzyme antagonists are thought to be present in the fish. Thiamine in large doses will prevent Chastek paralysis. In addition to the central nervous system, histological changes occur in the heart and liver. In subacute necrotizing encephalomyelopathy, no significant lesions have been reported outside the nervous system. Chastek paralysis is mentioned here because of points of similarity to subacute necrotizing encephalomyelopathy and thus to suggest that an enzyme antagonist may possibly have some part in the aetiology of both diseases.

EXPERIMENTAL FACTORS Experimental evidence has accumulated in favour of a vitamin $B_{1}$ deficiency as the aetiological basis of Wernicke's disease. This evidence has been reviewed by Cruickshank (1950). Histologically similar lesions in the nervous system have been produced experimentally in pigeons (Alexander, 1939), dogs (Zimmerman, 1939), and rats (Prickett, 1934). Lesions similar in topographical distribution and histological characteristics can be produced when animals are fed a diet deficient in vitamin $B_{1}$, if a disproportionately large supply of other vitamins is offered. On an entirely vitamin-free diet, the lesions are only rarely of the Wernicke type (Alexander, 1940).

Lesions produced experimentally with certain quinoline compounds have histological resemblances to those found in subacute necrotizing encephalomyelopathy (Richter, 1949). The mechanism behind the production of this experimental lesion is not clear although enzyme antagonisms may be involved. Also, lesions present in acute experimental inanition in cats were found to have progressive vascular changes similar to those found in subacute necrotizing encephalomyelopathy (Ferraro and Roizin, 1942). Many newly formed capillaries showing hypertrophy and hyperplasia of the endothelial cells were found. The mammillary bodies were involved to a lesser extent than seen with Wernicke's encephalopathy. Other features of experimental inanition not seen in subacute necrotizing encephalomyelopathy or Wernicke's disease were widespread neuronal degeneration (Ferraro and Roizin, 1942).

In summary, the aetiology of this condition has not been determined by experimental methods.

We are grateful to Dr. O. T. Bailey for counsel and assistance in the preparation of this paper. Dr. W. W. Hurteau provided case 2.

\section{REFERENCES}

Alexander, L. (1939). Arch. Neurol. Psychiat. (Chic.), 42, 1172. (1940). Amer. J. Path., 16, 61.

Blackwood, W., McMenemey, W. H., Meyer, A., Norman, R. M. and Russell, D. S. (1963). Greenfield's Neuropathology, 2nd ed., p. 407.Williams and Wilkins, Baltimore. Arnold, London.

Campbell, A. C. P., and Biggart, J. H. (1939). J. Path. Bact., 48, 245.

- and Russell, W. R. (1941). Quart. J. Med., 10, 41

Cruickshank, E. K. (1950). Ibid., 9, 327.

Eiben, R. M., Dooley, J. P., and Stowe, S. M. (1965). Neurology (Minneap.) 15, 293.

Evans, C. A., Carlson, W. E., and Green, R. G. (1942). Amer. J. Path., 18, 79.

Feigin, I., and Wolf, A. (1954). J. Pediat., 45, 243.

Ferraro, A., and Roizin, L. (1942). J. Neuropath. exp. Neurol., 1, 81.

Ford, F. R. (1960). Diseases of the Nervous System in Infancy, Childhood and Adolescence, 4th ed., p. 407. Thomas, Springfield, Ill.

Leigh, D. (1951). J. Neurol. Neurosurg. Psychiat., 14, 216.

Lewis, A. J. (1962). Canad. med. Ass. J., 87, 1085.

Meyer, A. (1944). J. Neurol. Neurosurg. Psychiat., 7, 66.

Namiki, H. (1965). Arch. Neurol. (Chic.), 12, 98

Peterson, H. de C., and Alvord, E. C., Jr. (1964). Trans. Amer. neurol. Ass., 89, 104.

Prickett, C. O. (1934). Amer. J. Physiol., 107, 459.

Reye, R. D. K. (1960). J. Path. Bact., 79, 165.

Richter, R. B. (1949). J. Neuropath. exp. Neurol., 8, 155.

, (1957). Ibid., 16, 281.

Riggs, H. E., and Boles, R. S. (1944). Quart J. Stud. Alcohol, 5, 361.

Tanaka, T. (1934). Amer. J. Dis. Child., 47, 1286.

Tom, M. I., and Rewcastle, N. B. (1962). Neurology (Minneap.), $12,624$.

Ule, G. (1959). Virchows Arch. path. Anat., 332, 204.

Verhaart, W. J. C. (1938). Arch. Dis Childh., 13, 225.

Wernicke, C. (1882). Lehrbuch der Gehirnkrankheiten, vol. 2, p. 229. T. Fischer, Cassel and Berlin.

Zimmerman, H. M. (1939). Yale J. Biol. Med., 12, 23. 